\title{
La Ville entrelacs. Littérature, histoire, peinture
}

\section{Roberta Sapino}

\section{(2) OpenEdition \\ 10 Journals}

\section{Edizione digitale}

URL: https://journals.openedition.org/studifrancesi/47775

DOI: $10.4000 /$ studifrancesi. 47775

ISSN: 2421-5856

\section{Editore}

Rosenberg \& Sellier

\section{Edizione cartacea}

Data di pubblicazione: 1 décembre 2021

Paginazione: 660-661

ISSN: 0039-2944

\section{Notizia bibliografica digitale}

Roberta Sapino, «La Ville entrelacs. Littérature, histoire, peinture», Studi Francesi [Online], 195 (LXV |

III) | 2021, online dal 01 décembre 2021, consultato il 07 décembre 2022. URL: http://

journals.openedition.org/studifrancesi/47775 ; DOI: https://doi.org/10.4000/studifrancesi.47775

Questo documento è stato generato automaticamente il 7 décembre 2022.

\section{(c) $($ ) $(9)$}

Creative Commons - Attribuzione - Non commerciale - Non opere derivate 4.0 Internazionale - CC BYNC-ND 4.0

https://creativecommons.org/licenses/by-nc-nd/4.0/ 


\title{
La Ville entrelacs. Littérature, histoire, peinture
}

\author{
Roberta Sapino
}

\section{NOTIZIA}

La Ville entrelacs. Littérature, histoire, peinture, dir. M. DA CONCEIÇÃo COELHO FERREIRA, Ph. MEUNIER, R. zsChaChlitZ, Lyon, Presses universitaires de Lyon, 2021, 204 pp.

1 Adottando un approccio pluridisciplinare in cui la prospettiva storica si interseca con gli studi linguistici, letterari e artistici, il volume coordinato da Maria DA CONCEIÇÃo COELHO FERREIRA, Philippe MEUNIER e Ralf zSCHACHLITZ - tutti e tre studiosi affiliati all'Université Lumière Lyon 2 - investiga gli equilibri, le tensioni, le metamorfosi che nel corso dei secoli hanno contribuito da un lato a determinare le specificità proprie di molte città, e dall'altro a delineare la nozione, sempre mutevole, di spazio urbano. Consci che la città prende forma in un reticolo complesso di particolarità geografiche, elementi architettonici e costrutti culturali e sociali, i curatori scelgono l'immagine dell'entrelacs come metafora delle dinamiche urbane di ogni tipo: un'immagine che dal Perceval di Chrétien de Troyes è entrata nel lessico architettonico, per poi essere usata in ambiti diversi tra cui la calligrafia e la tessitura, e che proprio per la sua capacità di attraversare discipline e arti eterogenee ben si adatta a rappresentare l'intreccio di epoche, vite e narrazioni di cui la città si compone (Maria DA CONCEIÇÃo COELHO FERREIRA, Philippe MEUNIER, Ralf ZsCHACHLITZ, Introduction, pp. 5-14).

2 La prima sezione, intitolata «Les replis de l'histoire», si concentra su alcune «lectures subjectives» delle città in cambiamento ed è composta da soli due contributi. Nel primo, Michèle VIGNAUX osserva i timori provocati dalla trasformazione di Londra in una città moderna tra il Cinque e il Seicento alla luce delle più ampie inquietudini legate all'emergere della nozione di individuo. Se i commentatori dell'epoca tendevano a lamentare gli effetti di un progressivo ma apparentemente inesorabile sfilacciamento del tessuto sociale, l'autrice dipinge un quadro dai toni più sfumati in cui emergono 
forme di vita comunitaria nuove («Fleur de toutes les cités» ou monstre difforme? Les ambiguïtés de Londres aux XVI ${ }^{\mathrm{e}}$ et XVII ${ }^{\mathrm{e}}$ siècles, pp. 17-30). Nel secondo, Maria DA CONCEIÇÃO COELHO FERREIRA si concentra sull'autore brasiliano Luiz Ruffato: nel suo romanzo più celebre Eles eram muitos cavalos (2001), poi nei cinque volumi del ciclo Inferno provisório (2005-2011), l'autore ricostruisce la storia delle classi operaie brasiliane nella seconda metà del Novecento. La frammentazione dello spazio e del tempo e la costruzione di un reticolo di storie individuali, in cui gli emarginati trovano una voce, consente all'autore di restituire le tensioni di uno stato sconvolto da una modernizzazione tanto rapida quanto sregolata ( $«$ La ville - des cicatrices qui cartographient mon corps»: l'espace urbain dans l'œuvre de Luiz Ruffato, pp. 31-52).

3 I quattro contributi che compongono la sezione «Cartographie de la rencontre» hanno in comune l'attenzione per lo spostamento di persone, luoghi, occasioni di incontro all'interno del tessuto urbano. In La place Bellecour à Lyon au XIX siècle, un entrelacs au cœur des déplacements, Emmanuelle ROMANET-DA FONSECA mostra come nell'Ottocento le variazioni della piazza simbolo di Lione (la comparsa di bar più o meno eleganti e di una stazione di polizia, ad esempio) riflettano i mutamenti sociali della città e in particolare i tentativi di moralizzazione operati dall'alto. La piazza è allora un lieu-enjeu nel quale si intrecciano due concezioni opposte dello spazio urbano: quella che vede la piazza come il cuore di una città accessibile a tutti, in cui categorie sociali diverse si incontrano e amalgamano, e quella che, rivendicando l'idea di quartiere, la vede come un salotto riservato alla buona società dei dintorni (pp. 55-72). Un altro luogo simbolico, il mercato, è al centro dell'analisi di Ingeborg RABENSTEIN-MICHEL, che legge il romanzo austriaco Ohnehin (2004) come una riattualizzazione urbana dell'AntiHeimatroman - genere che denunciava gli aspetti più reazionari, violenti e ostili del folklore rurale, e la cui produttività sembrava esauritasi negli anni Novanta. Contrariamente all'immagine idealizzata di crocevia di culture e luogo di integrazione che gli è spesso attribuita, nel corso del romanzo il Naschmarkt di Vienna si rivela un polo di tensioni sociali, gerarchie e violenze: l'idillio urbano si rivela così un miraggio tanto quanto quello rurale (Rencontres, passages et tissages dans "Ohnehin" de Doron Rabinovici: le virage urbain de l'Anti-Heimatroman", pp. 73-86). Ancora in area germanofona sono collocati i due contributi che chiudono la sezione. Il primo è Berlin sous la République de Weimar: perspectives françaises sur la jeune métropole et ses habitants, di Lydia BöHMERT. René Crevel, Yvan Goll, Henri Béraud, Jean Giraudoux e Joseph Kessel sono solo alcuni degli autori francesi che hanno viaggiato o vissuto a Berlino tra la fine degli anni Venti e i primi anni Trenta del Novecento, i cui sguardi si intersecano a comporre un ritratto della città nel quale si sovrappongono epoche diverse ed emergono commistioni culturali svariate, ma in cui - come fa notare l'autrice - si nota anche l'interferenza di pregiudizi importanti (pp. 87-102). Nell'ultimo, firmato da Ralf zschaChLITZ, l'analisi delle modalità di spostamento di Franz Biberkopf nella Berlino di Berlin Alexanderplatz apre a una più ampia discussione del rapporto tra l'uomo e una città diventata una giungla incomprensibile e ostile, nonché dell'attività dello scrittore, collocata nella tensione tra pensiero sulla storia e istanza poetica. Le parole di Benjamin, Sebald e Baudelaire sono specchi che permettono allo studioso di mettere in rilievo come il testo di Döblin solleciti, a livello sia della produzione che della ricezione, il confronto con la contingenza e l'arbitrarietà: per questo, Berlin Alexanderplatz anticipa alcune idee post-strutturaliste e postmoderne (Déplacement et narration dans "Berlin Alexanderplatz" d'Alfred Döblin, pp. 103-122). 
4 La sezione «Entrelacs des voix et des regards» racchiude infine contributi in cui l'attenzione è rivolta principalmente allo sguardo che osserva la città e alla sua trasposizione letteraria e artistica. Laura NGUYEN si sofferma su Città del Messico, e in particolare sulla sua raffigurazione in testi di viaggio con diversi gradi di letterarietà prodotti da autori italiani e spagnoli che vi sono stati tra i primi anni Novanta e il 2011: Pino Cacucci, Francisco Solano, Paco Nadal, Suso Mourelo e Manuel Reinaldo Méndez. Fungono da contrappunto a questi sguardi dall'esterno alcune voci autoctone, tra cui quelle dello scrittore Carlos Fuentes, del giornalista, saggista e attivista Carlos Monsiváis e del ricercatore Jezreel Salazar. Riprendendo le categorie forgiate da Marc Augé, Nguyen osserva come Città del Messico sia una «ville mémoire», una «ville rencontre», ma anche una «ville fiction», una vera e propria città palinsesto entrata di diritto nel mito (Regards croisés sur la ville de Mexico: ville entrelacs dans l'encre des voyageurs, pp. 125-142). A seguire, Marie LAUREILLARD guida il lettore alla scoperta dell'opera di Zhou Jirong et Zeng Hao, due artisti visivi cinesi nati negli anni Sessanta e attivi oggi. Con linguaggi comparabili benché diversi - l'uno evoca gli scarti e i punti di contatto tra il presente e il passato in uno spazio urbano in cambiamento, l'altro esprime soprattutto l'alienazione dell'individuo immerso in un mondo di cui si perde la coerenza - , i due artisti propongono delle rappresentazioni di Pechino in cui si manifesta il ciclo vorticoso e apparentemente incessante di distruzione e ricostruzione su cui si basa lo sviluppo della città (Le dialogue des artistes Zhou Jirong et Zeng Hao avec la ville chinoise, pp. 143-162). Gli ultimi due contributi riportano l'attenzione sull'Europa, e in particolare su Parigi e Londra. Élisa VIOLETTE BERNARD adotta un approccio odonimico - fondato sulla rappresentazione grafica su una mappa delle ricorrenze dei nomi di luogo presenti nei diversi romanzi - per studiare le rappresentazioni dello spazio urbano nei romanzi pubblicati da Aragon nel periodo tra le due guerre. A seguito dell'analisi dettagliata di Le paysan de Paris e di Les beaux quartiers, l'autrice riprende la definizione di atmosfera di Peter Zumthor per riflettere sul valore poetico della città (Le Paris d'Aragon: pour une poétique des usages urbains, pp. 163-176). Infine, in La ville tissée au fil de la voix: circulation et redistribution dans la fiction de Graham Swift, Pascale tOLLANCE si sofferma su The Sweet Shop Owner, Last Orders e The Light of Day, pubblicati tra il 1980 e l'inizio degli anni Duemila, per mettere in luce un tratto caratteristico dell'opera di Graham Swift: indipendentemente dal fatto che siano narrati in prima o in terza persona, e che siano polifonici o meno, nei suoi romanzi i luoghi e le voci appaiono come elementi indissociabili. «En même temps que le lieu existe par la voix, la voix fait du personnage, ou de ce qu'il en reste, un lieu de transit de la parole, un espace bruissant de mots» commenta l'autrice, e questo contribuisce a inscrivere Swift in un rapporto di continuità e scarto rispetto al modernismo (pp. 177-190).

Chiude il volume la conclusione dalla curatrice Maria DA CONCEIÇÃo COELHO FERREIRA, che fornisce piste di lettura trasversali tra i vari contributi e così valorizza la coerenza globale di questa miscellanea allo stesso tempo vasta e compatta, arricchita da immagini non numerosissime, ma sempre significative (Conclusion, pp. 191-198). 